\title{
MINI-REVIEW
}

\section{FDG Accumulation and Tumor Biology}

\author{
E. K. J. Pauwels, ${ }^{1,2}$ M. J. Ribeiro, ${ }^{2,3}$ J. H. M. B. Stoot,${ }^{1}$ V. R. McCready, ${ }^{4}$ \\ M. Bourguignon ${ }^{5}$ and B. Mazière ${ }^{2}$ \\ ${ }^{1}$ LEIDEN UNIVERSITY MEDICAL CENTRE, DEPARTMENT OF RADIOLOGY (DIVISION OF NUCLEAR MEDICINE), LEIDEN, THE \\ NETHERLANDS; ${ }^{2}$ SERVICE HOSPITALIER FRÉDÉRIC JOLIOT, DEPARTEMENT DE RECHERCHE MEDICALE, CEA, ORSAY, FRANCE; \\ ${ }^{3}$ FACULDADE DE MEDICINA UNIVERSIDADE DE COIMBRA, SERVIÇO DE MEDICINA, COIMBRA, PORTUGAL; ${ }^{4}$ THE ROYAL \\ MARSDEN HOSPITAL, DEPARTMENT OF NUCLEAR MEDICINE, SUTTON, SURREY, UK; AND ${ }^{5}$ OFFICE DE PROTECTION \\ CONTRE LES RAYONNEMENTS IONISANTS, LE VÉSINET, FRANCE
}

\begin{abstract}
The tumoral uptake of fluorine-18-deoxyglucose (FDG) is based upon enhanced glycolysis. Following injection, FDG is phosphorylated and trapped intracellularly. An important mechanism to transport FDG into the transformed cell is based upon the action of glucose transporter proteins; furthermore, highly active hexokinase bound to tumor mitochondria helps to trap FDG into the cell. In addition, enhanced FDG uptake may be due to relative hypoxia in tumor masses, which activates the anaerobic glycolytic pathway. In spite of these processes, FDG uptake is relatively aspecific since all living cells need glucose. Clinical use is therefore recommended in carefully selected patients. NUCL MED BIOL 25;4:317-322, 1998. (C) 1998 Elsevier Science Inc.
\end{abstract}

KEY WORDS. Scintigraphy, Fluorodeoxyglucose, Tumor biology

\section{INTRODUCTION}

Fluorine-18 deoxyglucose (FDG) is used by many centers for tumor imaging and assessment of the metabolic state of malignant lesions. There is ample evidence that the accumulation of FDG is based on enhanced glycolysis, which has often been associated with the growth rate and the malignancy potential of the tumor. Although many patients have benefited from scintigraphy with FDG, one should be aware of the diagnostic limitations associated with the use of this tracer. A greater understanding of this behaviour not only serves a scientific goal but it also helps to understand scintigraphic patterns observed under clinical circumstances in a better way.

To this end, this article reviews two major aspects of FDGscintigraphy: First, it provides an overview of tumor biology associated with glucose metabolism; second, it relates the relevant biological facts to characteristics of FDG in clinical tumor-scanning.

\section{ON THE ORIGIN OF CANCER}

How cancer arises is no longer a secret. According to modern insights, a tumor consists of cells in which a transformation has taken place owing to exposure to environmental factors, which may cause damage or alteration of the cellular DNA. Such factors include viruses, bacteria, parasites, a variety of chemicals and both ultraviolet and ionizing radiation. Among these carcinogenic agents, two important types have been recognized. First are those agents that damage genes or parts of genes that control cell proliferation and migration, and second are those agents that promote the growth of tumor cells or their precursor cells. In general, carcinogens cause specific cell functions to disappear and

Address correspondence to: Prof. Dr. E. K. J. Pauwels, Leiden University Medical Centre, Division of Nuclear Medicine, P.O. Box 9600, 2300 RC Leiden, The Netherlands.

Received 25 October 1997.

Accepted 26 November 1997. new functions to develop. Also, cell morphology may change, and the more the morphological grade declines during the transformation process the more the cell develops different characteristics, such as altered membrane properties, cell-to-cell interactions, and cell metabolism.

\section{Gene Alterations}

Two classes of genes play a major role in carcinogenesis: protooncogenes and suppressor genes. The first class of genes encourages cell enlargement and division, whereas the second can inhibit this proliferation. After a gene mutation, proto-oncogenes may become oncogenes, which are carcinogenic, leading to excessive multiplication due to uncontrolled overproduction of growth-stimulating proteins. Such proteins are involved in the pathways transducing growth signals from specific transporters on the outside of the cell through the cytoplasm to the nucleus. One of the most well-known oncogene is the ras oncogene, which produces proteins present in about a quarter of all human cancers $(7,40)$.

Tumor-suppressor genes counter the action of oncogenes and protect the organism from uncontrolled undifferentiated growth. Once mutated these suppressor genes may no longer produce proteins that inhibit this excessive cell growth. Therefore, inherited mutations in suppressor genes bring about a high cancer risk (52). The inactivated suppressor gene does not stop abnormal cell growth and a tumor may arise. The p53 gene on chromosome 17 is a suppressor gene that encodes for a protein able to inactivate ras and viral oncogenic proteins, thus reducing the probability that a normal cell transforms into a malignant cell (14). In certain families with high occurrence of breast carcinoma, a correlation with hereditary mutations of the $\mathrm{p} 53$ gene has been demonstrated (19). Recently, a direct relationship was shown between lung cancer and p53 mutations stemming from carcinogens cigarette smoke contained in (13). 


\section{Characteristics of Tumor Cells}

Tumor cells show a continuous multiplication, even when normal cells are at rest. They need less growth factor than do normal cells to achieve the same level of growth rate, and some tumor cells even have autocrine stimulation, producing both growth factors and their transporters. With higher growth rates, more mitoses are present, and the number of cells being in the S-phase will be relatively higher. With more cells in the S-phase the tissue needs more material to produce DNA.

Compared to a normal cell, a tumor cell is more rounded, with fewer attachments to the substratum and to other cells. Malignant cells may have distinct alterations in their surface protein, which promotes detachment from the original location. Thus, they may invade surrounding tissue and migrate to other, more remote tissues, establishing metastases. An example of an adhesion molecule that plays a role in the ability of the tumor cell to detach is E-cadherin (39).

Another feature of cancer cells is that they do not exhibit anchorage dependence. Normal cells need to adhere to the extracellular matrix in order to proliferate, and certain surface proteins (integrins) are involved in this attachment. If normal cells detach from the matrix, proliferation ceases and cell death occurs (apoptosis). Tumor cells do not exhibit this suicidal behaviour. They maintain their function, staying viable in circulation (39).

Transformed cells have fewer differentiated membranes and the membrane transporters are also less differentiated. This phenomenon is well-known in breast-cancer patients whose response to hormonal treatment diminishes with altered estrogen transporters (25). In addition, it appears that perfusion varies largely throughout the tumor. Its continuous growth, radially from the center, causes a higher perfusion in the dividing cells in the outer parts of the tumor bulk than in the core. This inner part is relatively hypoxic, which may result in necrotic areas and a virtually nonperfused necrotic mass. Also, there are intermittent periods of decreased flow alternated with resumption of flow, sometimes even in the opposite direction. These facts result in a very heterogeneous (vascular) morphology.

\section{GLUCOSE UPTAKE IN TUMOR CELLS}

Glucose assumes a central role among the fuels for cellular energy metabolism. Warburg was among the first to demonstrate that tumor cells have an altered glucose metabolism (48). Even under aerobic conditions these cells showed a high excretion of lactic acid. It was therefore concluded that the energy production of tumor cells occurs via two mechanisms: both the degradation of glucose to lactic acid and the oxidation of glucose (49). This increased glucose metabolism was primarily thought to be the result of increased glucose uptake and, in fact, this altered uptake was demonstrated by Hatanaka in experiments with murine sarcoma virus-infected mouse cells (22). Unexpectedly, the rate of glucose metabolism appeared not to be directly related to the glucose transport across the cell membrane.

In the same series of experiments, Hatanaka (22) used transforming cells induced by a Rous sarcoma virus infection or a murine sarcoma virus infection and demonstrated that an increased glucose transport over the cell membrane occurred even with non-metabolisable sugars such as 2-deoxyglucose (DG). From these experiments it was concluded that the increase in glucose uptake was due to either an alteration in the glucose transport system or an alteration in the functional nature of the membrane of transformed cells.

An elegant experiment by the same author, described in the same article (22), showed that cytochalasin B (an agent that inhibits glucose transport across the cell membrane) did not block the glucose transport in transformed cells. Hence, it was concluded that the membrane of the transformed cells allowed "infiltration" of glucose by a mechanism other than the initial sugar transport. This "infiltration" was considered characteristic for a transformed membrane.

The altered membrane characteristics were once more demonstrated by Martineau et al. (27). In a study with cultured normal cells it was observed that, even during growth, a high concentration of glucose in the medium had a "repressive" effect on the transport system and that a low concentration resulted in a "derepression" of glucose transport. In transformed cells, it appeared, however, that high sugar concentrations had no repressive effect on sugar transport, whereas the rates of both glucose consumption and lactic acid production were not higher than in a control group of cells (22).

\section{Glucose Transporters}

Insulin causes muscle and fat cells to absorb glucose from the circulation. This pancreas-produced hormone interacts with special transporters located in the cell membrane. These transporters are special proteins that help to convey glucose into the cell; therefore, they are called glucose transporters. These molecules have been thoroughly studied in human erythrocytes and are identified as heterogeneously glycosylated integral membrane proteins (30). There is sufficient evidence to show that the glucose molecule in circulation binds to the outer part of the transporter protein on the outside of the cell membrane, after which the complex changes conformation so as to move the glucose molecule to the inside of the membrane.

In the next step of this stereospecific process, the glucose molecule is released into the cytoplasm, and the transporter molecule resumes its original conformation in the membrane (Fig. 1). This transport of glucose involves hydrogen bonds between the glucose molecule and active parts of the transporter molecule, which "push" the glucose in the conveying process from the extracellular to the intracellular space (10). This stereospecific diffusion does not require energy, except in the case of the transporter that is abundant in the small intestine and the kidneys. This transporter is different from transporter molecules in other parts of the body in that it has a special affinity for fructose (24). This oscillation process moves glucose through the cell membrane from the high to the low glucose concentration, and in the erythrocyte the oscillation rate is about 900 times per second (1). It needs to be mentioned that this transport mechanism is based on the molecular structure of GLUT 1, which is the only protein that could be analysed properly because it has been isolated without functional loss. The transport mechanism as illustrated in Figure 1 is based on two different conformations of the transporter protein. Recently, the transport behaviour of GLUT 1 with alternative sugar import and sugar export was questioned (11).

So far, seven glucose transporters (GLUT 1-7) have been found, all consisting of a similar polypeptide chain of 500 amino acids with similar folding pattern $(3,4)$. These transporter isoforms are distributed over various tissues: GLUT 1 is abundant in erythrocytes, GLUT 2 in enterocytes and liver cells, GLUT 3 in the brain, GLUT 4 in skeletal and heart muscle, and GLUT 5 in the small 


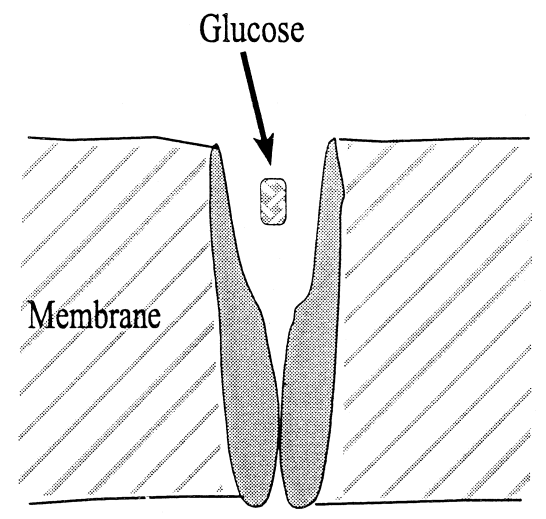

Cytosol

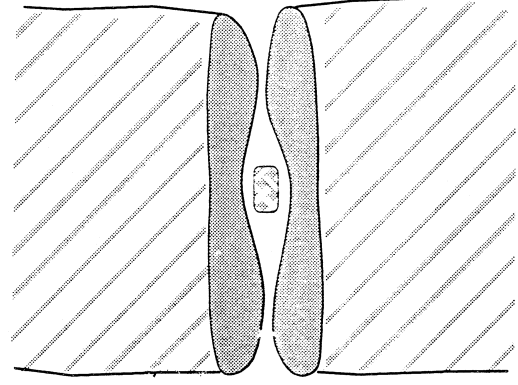

II

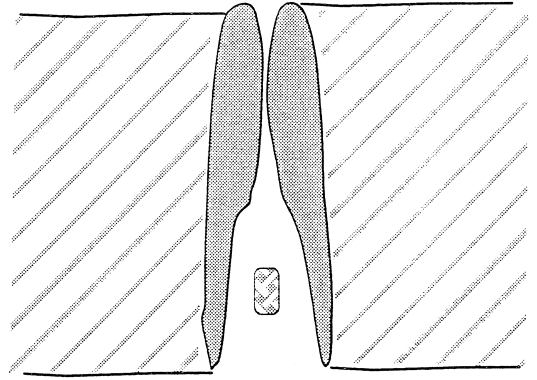

III

FIG. 1. Model for alternative conformation of the glucose transporter according to Carruthers et al. (10). Stages I-III show the import and export of the sugar molecule via the conveying glucose transporter from the extracellular to the intracellular space.

intestine and kidney (31). GLUT 6 is nonfunctional and GLUT 7 has been found mainly in hepatocytes $(3,4,31)$.

To reiterate, tumor cells display increased rate of glucose uptake as compared to untransformed cells, and the question arises whether this is due to increased activity of the glucose transporters, an increased biosynthesis of these molecules, or to the generation of a new class of transporters. Experiments performed about a decade ago revealed that cellular transformation coincides with elevated levels of glucose transporter messenger RNA $(6,17,23)$.

In cultured fibroblasts transfected with $s r c$ and ras oncogenes, it was found that glucose uptake was increased, paralleled by an increase in the amount of glucose transporter protein. In contrast, in cells transfected with the myc-oncogene, the glucose uptake was unchanged, which gives rise to the hypothesis that the origin of the tumor plays an important role (17). Thus, there is experimental evidence that malignant transformation results in an increased expression of the gene encoding for the glucose transport system.

The transcription of GLUT 1 and GLUT 3 is at a higher level in cancerous tissue than in normal tissue, and it has been suggested that overexpression of GLUT 1 and GLUT 3 genes is responsible for the increased uptake of glucose in malignancies $(32,53)$. Some confirmation of this hypothesis has been found by the increased expression of GLUT 1 and GLUT 3 in hepatocellular carcinoma and several brain tumors $(33,46)$.

\section{TRAPPING AND METABOLISM OF GLUCOSE AND DEOXYGLUCOSE}

When glucose enters the living cell, phosphorylation catalyzed by hexokinase occurs. The resulting molecule glucose-6-phosphate may enter further metabolic pathways. Glucose-6-phosphate inhibits hexokinase in an allosterical way, so that the uptake of glucose by cells is dependent on the rate by which glucose-6-phosphate is metabolized. The enzyme glucose- 6 -phosphate isomerase transfers glucose-6-phosphate into fructose-6-phosphate. This isomerization converts the aldose into a ketone through a cis-enediol intermediate. In effect, this is a rearrangement of the carbonyl group from the C-1 to the $\mathrm{C}-2$ position in the ring structure.

As with glucose, 2-deoxyglucose enters the cell and is phosphorylated by hexokinase to 2-deoxyglucose-6-phosphate. Further catabolysis of 2-deoxyglucose-6-phosphate is not possible as this would require the presence of an oxygen atom on the C-2 position.
Thus, 2-deoxyglucose-6-phosphate is trapped in the cell and, owing to a very low concentration of glucose-6-phosphate, the reverse reaction does not take place. Because of these characteristics, 2-deoxyglucose can be used to investigate glucose uptake of the cell.

The increased glucose metabolism of tumor cells has been suggested to be due to an increased activity of glycolytic enzymes $(50,51)$. In 1978 Monakhov et al. (29) reported on the fact that hexokinase played an important role in this process. It was found that in human malignant tissue the slower isoenzymes in the hexokinase spectrum showed a much lower abundance. Moreover, the same authors observed that the isoenzymes in tumor tissue showed an increased affinity for glucose.

Experiments by Bustamante et al. (9) with rat cells demonstrated that the cytoplasm of Ehrlich ascites tumor cells showed a high glycolytic rate compared to cytoplasm from normal liver tissue. When the mitochondria were extracted from the cytoplasm of the tumor cell, the aerobic glycolysis decreased, which illustrates the association of mitochondria with hexokinase. Furthermore, the rate of glycolysis of normal liver cytoplasm increased after the addition of tumor mitochondria, but not after the addition of normal mitochondria. In these experiments it also appeared that the addition of normal mitochondria to the tumor cytosol resulted in a glycolytic rate equal to that of normal cells. Also, it appeared that the addition of hexokinase extracted from tumor mitochondria resulted in an enhanced glycolytic rate.

These experiments suggest that highly active hexokinase bound to tumor mitochondria- a phenomenon not present in normal cells-causes the high glycolytic rate in tumor cells (2). Experiments by Pulselli et al. (36) have confirmed the special nature of hexokinase bound to the mitochondrial membrane of tumor cells. Moreover, Fanciulli et al. (15) observed both enhanced glycolytic and growth rates in transfected cells and ascribed this to the relatively high hexokinase activity as a consequence of both a high cellular metabolic demand and a more intense mitotic activity.

\section{SCINTIGRAPHY WITH FDG IN ONCOLOGY FDG Uptake in Relation to Pathophysiology}

Local cerebral glucose consumption was measured by Sokoloff et al. (43) in 1977 using carbon-14-labelled deoxyglucose in rat. One year later Gallagher et al. (18) reported on the use of ${ }^{18} \mathrm{~F}$-2-deoxy-2fluoroglucose (FDG) to study overall glucose metabolism in rodents. 
Soon after that, tumor detection in animals with FDG was achieved by Som et al. (44). The scintigraphic visualization of liver metastases in patients was described by Yonekura et al. (54), and Patronas et al. (34) reported on FDG accumulation in brain tumors and its relation to tumor necrosis. Since then numerous articles have appeared on the clinical use of FDG in oncology, and readers are referred to recent papers by Conti et al. (12), Rigo et al. (38), and Biersack et al. (5), discussing results in various cancers and clinical indications.

The fact that all living cells in the human body consume glucose interferes with the visualization of tumor tissue using FDG. High uptake is seen in those areas with low cellular glucose-6-phosphatase such as the brain and the heart. In contrast, the liver and the gut contain higher glucose-6-phosphatase levels, and in these organs the FDG uptake is relatively low (8). The FDG uptake in muscles is usually prominent, and this has led investigators to prescribe complete rest for patients in order to avoid muscle artifacts (38). Fasting, and thus hypoinsulinemia, reduces the availability of glucose transporters and may help to lower FDG uptake in muscles.

As indicated in this report, tumor tissue contains an increased amount of glucose transporter protein, and this phenomenon facilitates tumor detectability under fasting conditions with relative low uptake in the skeletal and cardiac muscle, by virtue of a higher tumor-to-nontumor ratio. It has also been suggested that high glucose plasma levels make FDG compete with glucose, resulting in a decreased FDG accumulation in neoplastic cells (47).

Among other biological phenomena that play a part in FDG accumulation in tumor cells, it is worthwhile to mention the increased transcription of GLUT 1 and GLUT 3 together with the presence of a highly active hexokinase isoform. This certainly helps to enhance the FDG uptake in the tumor. In addition, relative hypoxia, which often occurs in tumor masses, may activate the metabolic steps in the anaerobic glycolytic pathway and enhance FDG uptake (28), thereby contributing to an enhanced tumor-tonontumor ratio. In contrast, necrotic masses, which may be present in the same tumor, reverse this enhanced ratio.

It is hardly conceivable that the processes mentioned above occur in a controlled manner, and inter- and intra-individual cell-to-cell variations in tumor biochemistry are therefore likely. For clinical studies, this means that tumor FDG uptake is influenced by biochemical phenomena of which we have only fragmentary knowledge. It also explains the broad ranges between which FDG uptake varies (5).

Nevertheless, within the scope of this report it needs to be mentioned that FDG uptake has a high sensitivity (up to 90\%) to depict primary and secondary malignant lesions, but it has an imperfect specificity most likely due to the nonselective uptake mechanism. It is obvious that this radiopharmaceutical should therefore preferably be used in carefully selected patients. Also, one must be careful to adapt data on the accuracy of FDG scintigraphy without question as many articles mention sensitivity and specificity for lesion detection without clarifying in which way this was verified.

\section{Limitations of FDG Scintigraphy in Relation to Pathophysiology}

The use of FDG has often been advocated to study tumor viability. In its ongoing effort to image regional biochemistry, nuclear medicine should offer the potential to distinguish viable from nonviable cancerous tissue. Does FDG measure the functional status of a malignant lesion? The answer is definitely yes, but not without some critical comments. The radiopharmaceutical concentrates in metabolically active cells, thus distinguishing these from the less active ones. As already stated, tumor cells show higher glucose demand and can thus be differentiated scintigraphically from the cells with less high cellular turnover.

However, tumor tissue is nonhomogeneous. First, there is a scattered distribution of malignant tissue within the tumor. No tumor has a homogeneous tissue structure; clusters of normal cells alternate with clusters of (pre)malignant cells. This phenomenon occurs at a microscopic scale far beyond the resolution of the gamma camera. Consequently, scintigraphy with FDG measures an average energy demand in the tumor that does not fully reflect the metabolic status of the malignancy. This may be especially confusing if one wants to evaluate the effect of therapy.

Second, as a result of repair processes induced by physiological reactions or after tumor therapy, macrophages will replace tumor cells, these cells will also demonstrate FDG uptake. It has even been demonstrated that these macrophages show a higher FDG uptake than do viable tumor cells.

The confusing FDG uptake in the inflammatory response to tumor cells has been studied by Kubota et al. (26), who demonstrated that surrounding macrophages and newly formed granulation tissue showed greater FDG uptake than did viable tumor cells. A maximum of about $25 \%$ of the total ${ }^{18} \mathrm{~F}$ activity measured in tumor tissue was derived from macrophages and granulation tissues. The clinical consequence of this has been reported by Haberkorn et al. (20), who studied patients with colorectal tumors after radiotherapy. Secondary inflammation following therapy induced falsepositive FDG accumulation in these tumors, thus hampering the scintigraphic evaluation of therapy.

Third, literature on the time-dependent behaviour of FDG in tumors is very scarce. The uptake with time is most likely dependent on local parameters as mitotic activity and growth rate. Therefore, the interval between FDG administration and scintigraphy may affect the measured uptake. It may also explain the observed variation in uptake in apparently similar tumors in different patients (21).

Fourth, compartmental modelling requires a quantitative approach, and rate constants for a three-compartment model for 2 -deoxyglucose were derived from measurements in an animal model (43). The model has been validated in humans for FDG by Phelps et al. (35) in 1979 by measuring the cerebral glucose metabolic rate. The application of kinetic models assumes that radioactivity is spread homogeneously over the tumor, which is, as stated, rarely the case in solid tumors. This false assumption has thrown some doubts on the validity of the method (41).

To relate the quantitative kinetic behaviour of FDG to glucose, the lumped constant was developed and defined as the ratio of the FDG phosphorylation rate to the glucose phosphorylation rate under steady-state condition (37). This lumped constant appeared not to be influenced by plasma glucose levels in the rat (42), but may change during tumor treatment owing to physiological and biochemical response. This was exemplified by Fischman and Alpert (16), who elucidated the role of lysosomal acid hydrolases, released in the process of tumor treatment and the development of necrosis, causing further metabolism of glucose-6-phosphate. Also, hexokinase is a major factor in the transport characteristics of FDG and glucose. The relative activity of the various isoforms of hexokinase changes during treatment, causing altered phosphorylation rates for FDG and glucose. These kinetic changes influence the lumped constant and affect the evaluation of serial FDG uptake in patients undergoing treatment. 


\section{CONCLUDING REMARKS}

Although a large number of articles have appeared on the clinical use of FDG, only a few have paid attention to biological factors influencing the uptake of this radiopharmaceutical $(8,16,45)$. There is no doubt that with increasing cellular transformation, the malignant cell demands more glucose, and its requirements can be met by the upregulation of the membrane glucose transporter proteins. Also, the activation of enzymes involved in glucose metabolism and the deletion of nonrelevant enzymes may help the malignant cell to obtain the energy needed for proliferation.

Finally, it is important for nuclear medicine that FDG follows the initial biochemical route of glucose. Despite its limitations, FDG has served many clinical purposes and has helped to solve many individual clinical problems. An area that deserves special attention for future studies is the quantification of in vivo uptake of FDG. To study this, the timing, dietary conditions, and resting protocols of patients need to be standardized.

The authors are grateful to Ms. Tilly Hagendoorn for excellent secretarial assistance and to Dr. Tarek Abdelfatah el-Maghraby for supplying Figure 1. This work was supported by a grant of Le Ministère de l'Education Nationale, de l'Enseignement Supérieure et de la Recherche to E.K.J.P. under reference number DRIC/MDLM/MT 1701.

\section{References}

1. Appleman J. R. and Lienhard G. E. (1989) Kinetics of the purified glucose transporter: Direct measurement of the rates of interconversion of transporter conformers. Biochemistry 28, 8221-8227.

2. Arora K. K., Fancuilli M. and Pedersen P. L. (1990) Glucose phosphorylation in tumor cells: Cloning, sequencing, and overexpression in active form of a full-length cDNA encoding a mitochondrial bindable form of hexokinase. J. Biol. Chem. 265, 6481-6488.

3. Baldwin S. A., Barros L. F. and Griffith M. (1995) Trafficking of glucose transporters-signals and mechanisms. Biosci. Rep. 15, 419-426.

4. Bell G. I., Burant C. F., Takeda J. and Gould G. W. (1993) Structure and function of mammalian facilitative sugar transporters. J. Biol. Chem. 268, 19161-19164.

5. Biersack H. J., Bender H., Ruhlmann J., Schomburg A. and Grünwald F. (1997) FDG-PET in clinical oncology: Review and evaluation of results of a private clinical PET center. In: Nuclear Medicine Annual (Edited by Freeman L. M.) pp. 1-29. Lippincott-Raven Publishers, Philadelphia.

6. Birnbaum M. J., Haspel H. C. and Rosen O. M. (1987) Transformation of rat fibroblasts by FSV rapidly increases glucose transporter gene transcription. Science 235, 1495-1497.

7. Bourne H. R., Sanders D. A. and McCormick F. (1990) The GTPase superfamily: A conserved switch for diverse cell functions. Nature 348, 125-132.

8. Brock C. S., Meikle S. R. and Price P. (1997) Does fluorine-18 fluorodeoxyglucose metabolic imaging of tumours benefit oncology? Eur. J. Nucl. Med. 24, 691-705.

9. Bustamante E., Morris H. P. and Pedersen P. L. (1981) Energy metabolism of tumor cells: Requirement for a form of hexokinase with a propensity for mitochondrial binding. J. Biol. Chem. 256, 8699-8704.

10. Carruthers A. (1990) Facilitated diffusion of glucose. Physiol. Rev. 70, $1135-1175$.

11. Cloherty E. K., Heard K. S. and Carruthers A. (1996) Human erythrocyte sugar transport is incompatible with available carrier models. Biochemistry 35, 10411-10421.

12. Conti P. S., Liliern D. L., Hawley K., Keppler J., Grafton S. T. and Bading J. R. (1996) PET and $\left[{ }^{18} \mathrm{~F}\right]-\mathrm{FDG}$ in oncology: A clinical update. Nucl. Med. Biol. 23, 717-735.

13. Denissenko M. F., Pao A., Tang M. and Pfeifer G. P. (1996) Preferential formation of benzo[a]pyrene adducts at lung cancer mutational hot spots in p53. Science 274, 430-432.

14. el-Deiry W. S., Tokino T., Velculescu V. E., Levy D. B., Parsons R., Trent J. M., Lin D., Mercer W. E., Kinzler K. W. and Vogelstein B. (1993) WAF1, a potential mediator of p53 tumor suppression. Cell 75, $817-825$.
15. Fanciulli M., Paggi M. G., Btuno T., Delcarlo C., Bonetto F., Gentile F. P. and Florida A. (1994) Glycolysis and growth rate in normal and in hexokinase transfected NIH 3T3 cells. Oncol. Res. 6, 405-409.

16. Fischman A. J. and Alpert N. M. (1993) FDG-PET in oncology: There's more to it than looking at pictures. J. Nucl. Med. 34, 6-11.

17. Flier J. S., Mueckler M. M., Usher P. and Lodish H. F. (1987) Elevated levels of glucose transport and transporter messenger RNA are induced by ras or src oncogenes. Science 235, 1492-1495.

18. Gallagher B. M., Fowler J. S., Gutterson N. I., MacGregor R. R., Wan C. N. and Wolf A. P. (1978) Metabolic trapping as a principle of radiopharmaceutical design: Some factors responsible for the biodistribution of [18-F] 2-deoxy-2-fluoro-D-glucose. J. Nucl. Med. 19, 11541161.

19. Glebov O. K., McKenzie K. E., White C. A. and Sukumar S. (1994) Frequent p53 gene mutations and novel alleles in familial breast cancer. Cancer Res. 54, 3703-3709.

20. Haberkorn U., Straus L. G., Dimitrakopoulou A., Engenhart R., Oberdorfer F., Ostertag H., Romahn J. and van Kaick G. (1991) PET studies of fluorodeoxyglucose metabolism in patients with recurrent colorectal tumors receiving radiotherapy. J. Nucl. Med. 32, 1485-1490.

21. Hamberg L. M., Hunter G. J., Alpert N. M., Choi N. C., Babich J. W. and Fischman A. J. (1994) The dose uptake ratio as an index of glucose metabolism: Useful parameter or oversimplification? J. Nucl. Med. 35, $1308-1312$

22. Hatanaka M. (1974) Transport of sugars in tumor cell membranes. Biochim. Biophys. Acta 355, 77-104.

23. Hiraki Y., Rosen O. M. and Birnbaum M. J. (1988) Growth factors rapidly induce expression of the glucose transporter gene. J. Biol. Chem. 263, 13655-13662.

24. Inukai K., Katagiri H., Takata K., Asano T., Anai M., Ishihara H., Nakazaki M., Kikuchi M., Yazaki Y. and Oka Y. (1995) Characterization of rat GLUT5 and functional analysis of chimeric proteins of GLUT1 glucose transporter and GLUT5 fructose transporter. Endocrinology 136, 4850-4857.

25. Karnik P. S., Kulkarni S., Liou X. P., Budd G. T. and Bukowski R. M. (1994) Estrogen-receptor mutations in tamoxifen-resistant breast cancer. Cancer Res. 54, 349-353.

26. Kubota R., Yamada S., Kubota K., Ishiwata K., Tamahashi N. and Ido T. (1992) Intramural distribution of fluorine-18-fluorodeoxyglucose in vivo: High accumulation in macrophages and granulation tissue studies by microautoradiography. J. Nucl. Med. 33, 1972-1980.

27. Martineau R., Kohlbacher M., Shaw S. N. and Amos H. (1972) Enhancement of hexose entry into chick fibroblasts by starvation: Differential effect on galactose and glucose. Proc. Natl. Acad. Sci. U. S. A. $69,3407-3411$

28. Minn H., Clavo A. C. and Wahl R. L. (1996) Influence of hypoxia on tracer accumulation in squamous cell carcinoma: In vitro evaluation for PET imaging. Nucl. Med. Biol. 23, 941-946.

29. Monakhov N. K., Neistadt E. L., Shavlovskii M. M., Shartsman A. L. and Neifakh S. A. (1978) Physicochemical properties and isoenzyme composition of hexokinase from normal and malignant human tissues. J. Natl. Cancer Inst. 61, 27-33.

30. Mueckler M., Caruso C., Baldwin S. A., Panico M., Allard W. J., Lienhard G. E. and Lodish H. (1985) Sequence and structure of a human glucose transporter. Science 229, 941-945.

31. Mueckler M. and Holman G. (1995) Homeostasis without a GLUT. Nature 377, 100-101.

32. Murakami T., Nishiyama T., Shirotani T., Shinohara Y., Kan M., Ishii K., Kanai F., Nakazuru F. and Ebina Y. (1992) Type 1 glucose transporter from the mouse which are responsive to serum, growth factor, and oncogenes. J. Biol. Chem. 267, 9300-9306.

33. Nishioka T., Oda Y., Seino Y., Yamamoto T., Inagaki N., Yano H., Imura H., Shigemoto R. and Kikuchi H. (1992) Distribution of glucose transporters in human brain tumors. Cancer Res. 52, 3972-3979.

34. Patronas N. J., Di Chiro G., Brooks R. A., DeLaPaz R. L., Kornblith P. L., Rizzoli H. V., Kessler R. M., Manning R. G., Channing M., Wolf A. P. and O'Connor C. M. (1982) Work in progress: $\left[{ }^{18} \mathrm{~F}\right]$ Fluorodeoxyglucose and positron emission tomography in the evaluation of radiation necrosis of the brain. Radiology 144, 885-889.

35. Phelps M. E., Huang S. C., Hoffman E. J., Selin C., Sokoloff L. and Kuhl D. E. (1979) Tomographic measurement of local cerebral glucose metabolic rate in humans with (F-18) 2-fluoro-2-deoxy-D-glucose: Validation of method. Ann. Neurol. 6, 371-388.

36. Pulselli R., Amadio L., Fanciulli M. and Floridi A. (1996) Effect of 
lonidamine on the mitochondrial potential in situ in Ehrlich ascites tumor cells. Anticancer Res. 16, 419-423.

37. Reivich M., Alavi A., Wolf A., Fowler J., Russell J., Arnett C., MacGregor R. R., Shine C. Y., Atkins H. and Anand A. (1985) Glucose metabolic rate kinetic model parameter determination in humans: The lumped constants and rate constants for $\left[{ }^{18} \mathrm{~F}\right]$ fluorodeoxyglucose and $\left[{ }^{11}\right.$ C]deoxyglucose. J. Cereb. Blood Flow Metab. 5, 179-192.

38. Rigo P., Paulus P., Kaschten B. J., Hustinx R., Bury T., Jerusalem G., Benoit T. and Foidart-Willems J. (1996) Oncological applications of positron emmission tomography with fluorine-18 fluorodeoxyglucose. Eur. J. Nucl. Med. 23, 1641-1674.

39. Ruoslahti E. and Reed J. C. (1994) Anchorage dependence, integrins and apoptosis. Cell 77, 477-478.

40. Scheffzek K., Ahmadian M. R., Kabsch W., Wiesmüller L., Lautwein A., Schmitz F. and Wittinghofer A. (1997) The ras-rasGAP complex: Structural basis for GTPase activation and its loss in oncogenic ras mutants. Science 277, 333-338.

41. Schmidt K. C., Lucignani G. and Sokoloff L. (1996) Fluorine-18fluorodeoxyglucose PET to determine regional cerebral glucose utilisation: A re-examination. J. Nucl. Med. 37, 394-399.

42. Schuier F., Orzi F., Suda S., Lucignani G., Kennedy C. and Sokoloff L. (1990) Influence of plasma glucose concentration on lumped constant of the deoxyglucose method: Effects of hyperglycemia in the rat. J. Cereb. Blood Flow Metab. 10, 765-773.

43. Sokoloff L., Reivich M., Kennedy C., Des Rosiers M. H., Patlak C. S., Pettygrew K. D., Sakurada O. and Shinohara M. (1977) The [14Cldeoxyglucose method for the measurement of local cerebral glucose utilisation: Theory, procedure, and normal values in the conscious and anesthetized albino rat. J. Neurochem. 28, 897-916.

44. Som P., Atkins H. L., Bandoypadhyay D., Fowler J. S., MacGregor R. R., Matsui K., Oster Z. H., Sacker D. F., Shiue C. Y., Turner H., Wan C.-N. and Zabinski S. V. (1980) A fluorinated glucose analog, 2-fluoro-
2-deoxy-D-glucose (F-18): Nontoxic tracer for rapid tumor detection. J. Nucl. Med. 21, 670-675.

45. Strauss L. G. (1996) Fluorine-18 deoxyglucose and false-positive results: A major problem in the diagnostics of oncological patients. Eur. J. Nucl. Med. 23, 1409-1415.

46. Su T.-S., Tsai T.-F., Chi C.-W., Han S.-H. and Chou C.-K. (1990) Elevation of facilitated glucose transporter messenger RNA in human hepatocellular carcinoma. Hepatology 11, 118-122.

47. Wahl R. L., Cody R. L., Hutchins G. D. and Mudgett E. E. (1991) Primary and metastatic breast carcinoma: Initial clinical evaluation with PET with the radiolabeled glucose analogue 2-[F-18]-fluoro-2deoxy-D-glucose. Radiology 179, 765-770.

48. Warburg O. (1925) Uber den Stoffwechsel der Carcinomzelle. Klin. Wochenschr. Berl. 4, 534-536.

49. Warburg O. (1956) On the origin of cancer cells. Science 123, 309-314

50. Weber G. (1977) Enzymology of cancer cells (first of two parts). N. Engl. J. Med. 296, 486-492.

51. Weber G. (1977) Enzymology of cancer cells (second of two parts). N. Engl. J. Med. 296, 541-551.

52. Wooster R., Bignell G., Lancaster J., Swift S., Seal S., Mangion J., Collins N., Gregory S., Gumbs C. and G. M. (1995) Identification of the breast cancer susceptibility gene BRCA2. Nature 378, 789-792.

53. Yamamoto T., Seino Y., Fukumoto H., Koh G., Yano H., Inagaki N., Yamada Y., Inoue K., Manabe T. and Imura H. (1990) Over-expression of facilitative glucose transporter genes in human cancer. Biochem. Biophys. Res. Commun. 170, 223-230.

54. Yonekura Y., Benua R. S., Brill A. B., Som P., Yeh S. D. J., Kemeny N. E., Fowler J. S., MacGregor R. R., Stamm R., Christman D. R. and Wolf A. P. (1982) Increased accumulation of 2-deoxy-2-[18F]-fluoroD-glucose in liver metastases from colon carcinoma. J. Nucl. Med. 23, 1133-1137. 